\title{
Ricordi di Gianfranco Folena
}

\author{
Lino Leonardi
}

PUBBLICATO: 31 MAGGIO 2021

$\mathrm{E}$ ra un pomeriggio d'autunno del 1986 quando incontrai Gianfranco Folena per la prima volta, nella sala del Circolo a Padova, lui presidente della commissione di ammissione al dottorato di ricerca, io tra i candidati alla prova scritta: poco più di cinque anni prima della sua scomparsa. Il mio contributo personale alla sua memoria può essere dunque poca cosa, a fronte di chi lo ha conosciuto e frequentato ben più a lungo e più da vicino. Se ho tuttavia accettato l'invito a prendere brevemente la parola al termine della Tornata Accademica dedicata dalla Crusca al centenario della sua nascita è appunto per portare una piccola testimonianza, dal punto di vista non scontato di chi conobbe Folena come un maestro, ma non da allievo diretto, venendo da un altro ambiente, e in quei suoi ultimi anni già presto sofferenti.

Fui ammesso al dottorato: era un'istituzione ancora recente, il terzo ciclo, e mi avevano preceduto li a Padova solo laureati padovani, allievi di Folena o della sua grande scuola. Ebbi chiara la sensazione, fin dai primi colloqui nel piccolo studio del Palazzo Maldura, tra le domande sui miei studi a Firenze e i saluti di cui mi incaricava per questo o quel suo antico compagno, spesso del tutto al di fuori della mia portata, ebbi la sensazione che per lui quell'ammissione di un fiorentino alla sua scuola padovana fosse come un ritorno, un modo per ritrovare le tracce del percorso da Firenze a Padova che era stato il suo tanti anni prima: certo allora - dopo la guerra e la prigionia, il comando alla Crusca il periodo di assistente universitario a Firenze - con una maturità e una consapevolezza incomparabili a quelle che potevano essere le mie.

Purtroppo a quell'accoglienza affettuosa e quasi complice non poté far seguito il consolidamento di un rapporto di discepolato che forse entrambi, io certo e fortemente, desideravamo: furono gli anni della sua malattia, che diradò ben presto le occasioni di incontro. Ricordo tuttavia ancora quando mi intrufolai a una sua lezione, sorpreso e affascinato da un'arte della divagazione che apriva scenari linguistici e letterari inimmaginabili dietro i pochi versi commentati, e poi confuso dal borbottio con cui mi invitò affettuosamente ma inequivocabilmente a non riprovarci. Ricordo soprattutto le conversazioni sul mio tema di ricerca, Guittone, e l'immediato suo coinvolgermi nella redazione dell'edizione delle lettere guittoniane che stava ultimando il suo vecchio amico Claude Margueron ${ }^{[\mathrm{I}]}$, segno tangibile per me alle prime armi della totale, impegnativa fiducia di cui era capace Folena, tale da suscitare competenze e dedizione che non credevi di possedere, nonché segno della sua incredibile rete di rapporti internazionali, che erano in primo luogo rapporti personali di amicizia. E ricordo la sua curiosità, e l'entusiasmo di consigli e stimoli, quando gli parlai dell'idea di lavorare, con un gruppo di amici, sulle traduzioni medievali della Bibbia in italiano, senza sapere che proprio in quel periodo stava aggiornando il suo saggio su Volgarizzare e tradurre, uno dei più memorabili e produttivi su uno dei temi a lui più cari ${ }^{[2]}$.

Ma ciò di cui posso e vorrei dare testimonianza non è il Folena maestro, di cui non oserei parlare. È invece l'ambiente che lui aveva creato intorno a sé, per come lo poteva percepire un neofita che veniva da Firenze. Quella fu per me davvero un'esperienza tanto inattesa quanto formativa, e credo emblematica della figura intellettuale di Folena e del ruolo che fu il suo nella società culturale non solo italiana del secondo Novecento. 
Ai miei occhi di nuovo arrivato, ma credo non solo ai miei, il momento più rappresentativo di quella comunità di studi erano le sedute del circolo, ogni mercoledi. Lo stesso Folena lo aveva descritto cosi, nel i966, pochi anni dopo averlo avviato nel ig63:

Il nostro circolo non vuole [...] né può vantare prerogative teoriche o metodologiche; ad esso noi siamo affezionati come al nostro particolare Zirkel im Verstehen, un confortante luogo d'incontro e di comprensione, fra la lettura individuale e la conversazione comune, in un'epoca incline al formalismo logico e all'astrazione spesso dogmatica, in cui rimane sempre meno tempo per leggere e per conversare, e mentre si attribuisce tanto rilievo al processo della «comunicazione», si finisce non di rado per perdere di vista l'oggetto della comunicazione, le cose e il valore delle cose ${ }^{[3]}$.

A parte l'accenno polemico alle teorie e alla scienza della comunicazione, che dà comunque la misura della totale indipendenza dal contesto allora dominante, traspare qui l'intento di ciò che Folena aveva creato, e che io ritrovai vent'anni dopo intatto e anzi perfezionato, con lo stesso lucido entusiasmo e le stesse coordinate culturali: lettura individuale e conversazione comune, confronto con i testi e condivisione dell'esperienza. Folena stesso, e poi tanti dopo di lui, hanno attribuito la fondazione del circolo padovano al tentativo di riprodurre quel circolo linguistico che Folena aveva frequentato nei suoi anni fiorentini. Ma la creatura di Folena era tutt'altra cosa, ci si respirava più l'eredità di Pasquali, intendo il Pasquali delle "pagine stravaganti", che non la linguistica di Devoto o Migliorini, cui pure era anche legatissimo e che praticava con sicurezza: di questo allargamento di prospettiva ovviamente era ben consapevole fin dall'inizio, come indicava simbolicamente anche soltanto l'aggiunta della filologia nel titolo del circolo, ma non so quanto potesse percepire la novità che quei pomeriggi rappresentavano per un dottorando come me, che mi ero affacciato da studente alle sedute fiorentine, e immagino per tanti altri prima e dopo di me.

A Padova il circolo era un appuntamento imprevedibile, che portava a confrontarsi con ogni aspetto della vita linguistica, filologica e letteraria d'Europa, dai Giuramenti di Strasburgo a Zanzotto, e quella straordinaria apertura di temi e di punti di vista lasciava intravedere le linee di forza della cultura e della ricerca di Folena, e via via dei suoi primi allievi, poi colleghi. Un'esperienza che inoltre, per non so quale segreta arte di Folena, sapeva farsi in effetti collettiva, sapeva coinvolgere e con ciò stesso creare il sentimento di un gruppo, di una comunità scientifica e letteraria ${ }^{[4]}$. E il momento per me più atteso e spettacolare di quelle sedute era, inevitabilmente, l'intervento di Folena: non quello introduttivo, le parole di rito con cui presentava il relatore di turno, spesso allievo o amico, giovane alle prime armi o illustre accademico, ma l'immancabile primo intervento che apriva la discussione alla fine della conferenza e che, qualsiasi fosse l'argomento, si dilungava a riprenderne i punti salienti, a ricostruire il contesto, a indicare possibili piste di sviluppo trascurate, ad aggiungere un anello mancante all'argomentazione, a contestare talvolta qualche tesi: a rifare insomma la conferenza, o meglio a suggerire per lo più benevolmente un altro modo di vedere le cose, più preciso spesso, sempre più ampio e ricco di armoniche, di spessore storiografico e di finezza interpretativa.

Sul circolo filologico-linguistico di Folena esiste già una bibliografia, e il mio non vuole essere altro che un ricordo, da parte di uno dei meno autorizzati tra i molti che hanno avuto la fortuna di assistervi. Condividerlo oggi in questa giornata di memorie significa provare a esprimere cio che ha rappresentato il suo magistero, certo non solo per me, al di là del valore dei suoi lavori filologici, linguistici, critici: la possibilità di una visione della letteratura come chiave della storia europea, di una visione della filologia e della linguistica come strumenti vivi di un confronto rigoroso e appassionato con i testi del passato e del presente, e insieme la necessità che il nostro lavoro fosse comprensibile e trovasse alla fine un senso solo attraverso una compartecipazione umana, un confronto autentico di personalità, una comunità intellettuale che fosse parte e avesse un ruolo nella società contemporanea. 
Di questo esempio soprattutto sono grato al magistero di Gianfranco Folena.

Note:

I. Guittone d'Arezzo, Lettere, edizione critica a cura di C. Margueron, Bologna, Commissione per i testi di lingua, I990.

2. G. Folena, Volgarizzare e tradurre, Torino, Einaudi, I99I (prima ed. «Volgarizzare» e «tradurre»: idea e terminologia della traduzione dal Medioevo italiano e romanzo all'Umanesimo europeo, in aa.vv., La traduzione, saggi e studi, Trieste, Lint, I973, pp. 57-I20).

3. G. Folena, Premessa, in Ricerche sulla lingua poetica contemporanea, Padova, Liviana, I966, p. ix.

4. Già nel r980 del resto Roncaglia aveva parlato dell'impegno di Folena "nell'organizzazione del lavoro collettivo": Au. Roncaglia, Lettera a Gianfranco Folena, in Studi di filologia romanza e italiana offerti a Gianfranco Folena dagli allievi padovani, Modena, Mucchi, r980, p. ix.

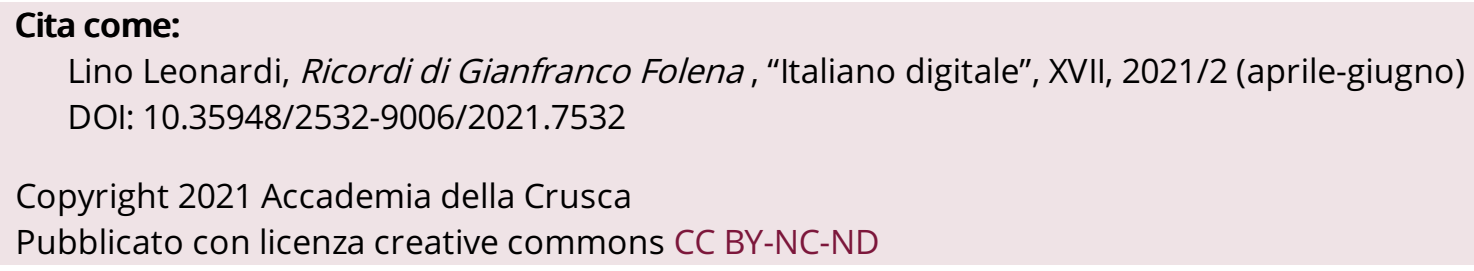

